\title{
Descent properties of hermitian Witt groups in inseparable extensions
}

Archiv der Mathematik Archives Mathématiques Archives of Mathematics

ISSN 0003-889X

Volume 96

Number 6

Arch. Math. (2011) 96:547-553

DOI 10.1007/

s00013-011-0261-2

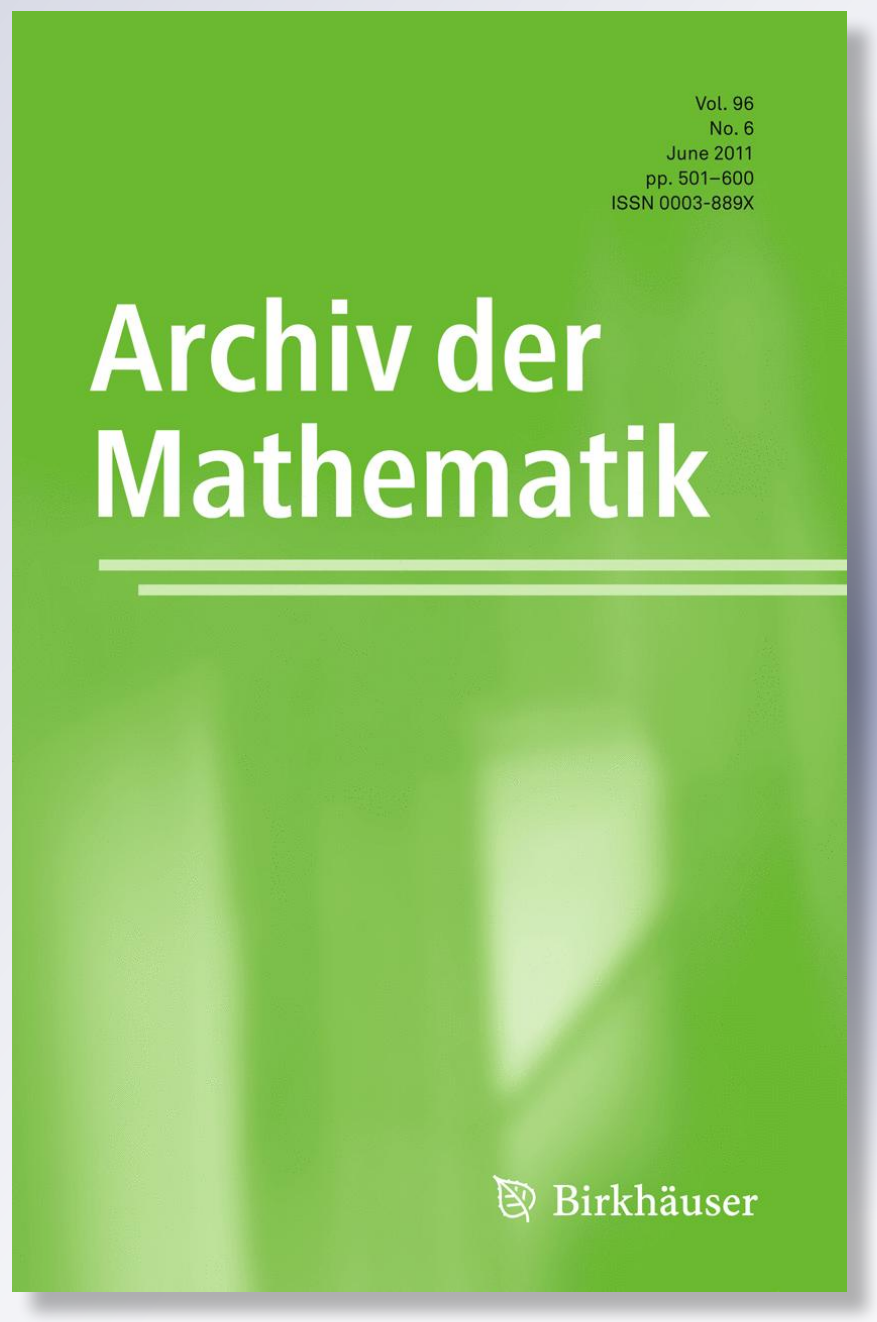

算 Springer 
Your article is protected by copyright and all rights are held exclusively by Springer Basel AG. This e-offprint is for personal use only and shall not be self-archived in electronic repositories. If you wish to self-archive your work, please use the accepted author's version for posting to your own website or your institution's repository. You may further deposit the accepted author's version on a funder's repository at a funder's request, provided it is not made publicly available until 12 months after publication. 


\title{
Descent properties of hermitian Witt groups in inseparable extensions
}

\author{
Eva Bayer-Fluckiger And Daniel Arnold Moldovan
}

\begin{abstract}
Let $k$ be a field of characteristic $\neq 2, A$ be a central simple algebra with involution $\sigma$ over $k$ and $W(A, \sigma)$ be the associated Witt group of hermitian forms. We prove that for all purely inseparable extensions $L$ of $k$, the canonical map $r_{L / k}: W(A, \sigma) \longrightarrow W\left(A_{L}, \sigma_{L}\right)$ is an isomorphism.
\end{abstract}

Mathematics Subject Classification (2010). 11E81.

Keywords. Hermitian forms, Witt groups, Central simple algebras with involution, Extension of scalars, Purely inseparable extensions.

1. Introduction. Let $k$ be a field of characteristic $\neq 2$ and let $W(k)$ denote the Witt ring of $k$. If $L / k$ is a field extension, we obtain a canonical ring homomorphism $r_{L / k}: W(k) \rightarrow W(L)$. By a classical theorem of Springer, this homomorphism is injective if the extension has odd degree. Moreover, Rosenberg and Ware prove $([7$, Theorem A] $)$ that if $L / k$ is Galois of odd degree, then $r_{L / k}: W(k) \rightarrow W(L)^{\operatorname{Gal}(L / k)}$ is an isomorphism. More generally, Rost describes ([8, Proposition 1]) the image of $r_{L / k}$ for any separable field extension of odd degree. When $L / k$ is purely inseparable, it is easy to see that $r_{L / k}: W(k) \rightarrow W(L)$ is bijective.

Let $A$ be a finite dimensional $k$-algebra with a $k$-linear involution $\sigma: A \rightarrow A$, and let $W(A, \sigma)$ be the associated Witt group (cf. Section 2). For any field extension $L / k$ there is a canonical group homomorphism $r_{L / k}: W(A, \sigma) \rightarrow$ $W\left(A_{L}, \sigma_{L}\right)$. Several of the above properties can be generalized. In particular, if $L / k$ has odd degree, then $r_{L / k}$ is injective (cf. [2, Proposition 2]), and analogues of the results of Rosenberg, Ware and of Rost are proved in [1].

D. A. Moldovan was partially supported by the Swiss National Science Foundation, grant 200020-109174/1. 
The aim of the present paper is to show the following:

Theorem. Let $(A, \sigma)$ be a central simple algebra with involution over $k$ and $L$ be a purely inseparable extension of $k$. Then the canonical homomorphism $r_{L / k}: W(A, \sigma) \rightarrow W\left(A_{L}, \sigma_{L}\right)$ is an isomorphism.

2. Definitions, notation and basic facts. Let $A$ be a finite dimensional simple $k$-algebra. An involution on $A$ is an anti-automorphism $\sigma: A \rightarrow A$ of order 2 . Let $K$ be the center of $A$. We say that $(A, \sigma)$ is a central simple algebra with involution over $k$ if the fixed field of $\sigma$ in $K$ is equal to $k$. If $K=k$, then $\sigma$ is said to be of the first kind. In this case, after extension to $k_{s}$, the involution $\sigma$ is determined by a symmetric or a skew-symmetric form. In the first case, $\sigma$ is said to be of the orthogonal type, whereas in the second one, of the symplectic type. If $K \neq k$, then $K$ is a quadratic extension of $k$ and the restriction of $\sigma$ to $K$ is the non-trivial automorphism of $K$ over $k$. In that case, the involution is said to be of the second kind, or a unitary involution, or a $K / k$-involution.

Basic results on central simple algebras with involution can be found for instance in $[6$, Chapter 1$]$

Let $(A, \sigma)$ be a finite dimensional algebra with involution over $k$, and let $\epsilon= \pm 1$. An $\epsilon$-hermitian form over $(A, \sigma)$ is by definition a pair $(V, h)$, where $V$ is a projective right $A$-module of finite rank, and $h: V \times V \rightarrow A$ is nondegenerate hermitian with respect to $\sigma$. We say that $(V, h)$ is hyperbolic if there exists a projective sub- $A$-module $W$ of $V$ with $\operatorname{dim}_{k}(V)=2 \operatorname{dim}_{k}(W)$ and such that $h(x, y)=0$ for all $x, y \in W$. This leads to a notion of Witt group $W^{\epsilon}(A, \sigma)$ (cf. for instance [9, Chapter 7, $\left.\S 2\right]$ or [5, Chapter 1, $\left.\S 10\right]$ ). Note that the tensor product of a quadratic form over $k$ with an $\epsilon$-hermitian form over $(A, \sigma)$ is an $\epsilon$-hermitian form over $(A, \sigma)$, hence $W^{\epsilon}(A, \sigma)$ is a $W(k)$-module.

If $L / k$ is a field extension, then we define $A_{L}=A \otimes_{k} L$ and the involution $\sigma_{L}=\sigma \otimes \operatorname{id}_{L}$ on $A_{L}$. We have a canonical homomorphism

$$
r_{L / k}: W^{\epsilon}(A, \sigma) \rightarrow W^{\epsilon}\left(A_{L}, \sigma_{L}\right) .
$$

We start by recalling two results that will be used in the sequel.

Theorem 2.1. Suppose that $L / k$ is a finite extension of odd degree. Then

$$
r_{L / k}: W^{\epsilon}(A, \sigma) \rightarrow W^{\epsilon}\left(A_{L}, \sigma_{L}\right)
$$

is injective.

Proof. See [2, Proposition 2 ]

Let $L$ and $L^{\prime}$ be two finite separable extensions of $k$. Set $R=L \otimes_{k} L^{\prime}$, and let $i: L \rightarrow R, i^{\prime}: L^{\prime} \rightarrow R$ be defined by $i(x)=x \otimes 1$ and $i^{\prime}\left(x^{\prime}\right)=1 \otimes x^{\prime}$. Let $A_{R}=A \otimes_{k} R$. The maps $i$ and $i^{\prime}$ induce

$$
j: W^{\epsilon}\left(A_{L}, \sigma_{L}\right) \rightarrow W^{\epsilon}\left(A_{R}, \sigma_{R}\right),
$$

respectively

$$
j^{\prime}: W^{\epsilon}\left(A_{L^{\prime}}, \sigma_{L^{\prime}}\right) \rightarrow W^{\epsilon}\left(A_{R}, \sigma_{R}\right) .
$$

In the special case when $L=L^{\prime}$, we get two maps

$$
j, j^{\prime}: W^{\epsilon}\left(A_{L}, \sigma_{L}\right) \rightarrow W^{\epsilon}\left(A_{R}, \sigma_{R}\right) .
$$


Theorem 2.2. Suppose that $L$ is a finite separable extension of odd degree of $k$. Then the sequence of $W(k)$-modules

$$
0 \rightarrow W^{\epsilon}(A, \sigma) \stackrel{r_{L / k}}{\longrightarrow} W^{\epsilon}\left(A_{L}, \sigma_{L}\right) \stackrel{j-j^{\prime}}{\longrightarrow} W^{\epsilon}\left(A_{R}, \sigma_{R}\right)
$$

is exact.

Proof. This is proved in [1, Corollary 3.2]. Note that the Witt groups considered in [1] are slightly different, but the proof of the result carries over without change to our situation.

3. An exact sequence. The aim of this section is to recall some results that will be used later. In particular, we need part of an exact sequence due to Grenier-Boley and Mahmoudi (cf. [4, Corollary 6.1], see also Appendix 2 of [3] for the initial exact sequence, due to Parimala, Sridharan, and Suresh). We start by introducing some notation and terminology. We keep the notation of the previous section, in particular $k$ is a field of characteristic $\neq 2$.

Let $(A, \sigma)$ be a finite dimensional central simple algebra with involution over $k$ and denote by $K$ the center of $A$.

We say that $(A, \sigma)$ satisfies hypothesis $(*)$ if we have the following:

(*) There exist $\lambda, \mu \in A^{*}$ such that $\sigma(\lambda)=-\lambda, \sigma(\mu)=-\mu, \mu \lambda=-\lambda \mu$ and $E=K(\lambda)$ is a quadratic extension of $K$.

Suppose that $(A, \sigma)$ satisfies hypothesis $(*)$ and let $\tilde{A}$ be the centralizer of $E$ in $A$. We then have $\mu \tilde{A} \mu^{-1}=\tilde{A}, \mu^{2} \in \tilde{A}, \sigma(\tilde{A})=\tilde{A}$ and $A=\tilde{A} \oplus \mu \tilde{A}$. We define two involutions on $\tilde{A}$ in the following way: let $\sigma_{1}=\left.\sigma\right|_{\tilde{A}}$ and $\sigma_{2}=\operatorname{Int}\left(\mu^{-1}\right) \circ \sigma_{1}$. There are two $E$-linear projections:

$$
\pi_{i}: A \rightarrow \tilde{A}, \pi_{i}\left(a_{1}+\mu a_{2}\right)=a_{i}, \quad i=1,2 .
$$

If $h: V \times V \rightarrow A$ is a $-\epsilon$-hermitian form over $(A, \sigma)$, we define the map

$$
h_{2}: V \times V \rightarrow \tilde{A}, \quad h_{2}(x, y)=\pi_{2}(h(x, y)) .
$$

Then $h_{2}$ is an $\epsilon$-hermitian form on $\left(\tilde{A}, \sigma_{2}\right)$ and the assignement $h \mapsto h_{2}$ induces a homomorphism

$$
\pi_{2}: W^{-\epsilon}(A, \sigma) \rightarrow W^{\epsilon}\left(\tilde{A}, \sigma_{2}\right) .
$$

We define a homomorphism

$$
\rho_{1}: W^{\epsilon}\left(\tilde{A}, \sigma_{1}\right) \rightarrow W^{-\epsilon}(A, \sigma)
$$

in the following way: if $f: V \times V \rightarrow \tilde{A}$ is an $\epsilon$-hermitian form on $\left(\tilde{A}, \sigma_{1}\right)$, then set $\rho(f)$ to be equal to $\lambda f$ on $V$ and extend it by sesquilinearity on $V \otimes V \mu=V \otimes_{\tilde{A}} A$.

We analogously define a homomorphism

$$
\rho_{2}: W^{\epsilon}\left(\tilde{A}, \sigma_{2}\right) \rightarrow W^{-\epsilon}(A, \sigma):
$$

if $f: V \times V \rightarrow \tilde{A}$ is an $\epsilon$-hermitian form on $\left(\tilde{A}, \sigma_{2}\right)$, then set $\rho(f)$ to be equal to $\lambda \mu f$ on $V$ and extend it by sesquilinearity on $V \otimes V \mu=V \otimes_{\tilde{A}} A$. It is easy to check that all of the above homomorphisms are well-defined. 
Theorem 3.1. Suppose that $(A, \sigma)$ satisfies hypothesis $\left(^{*}\right)$. Then the following sequence is exact

$$
W^{\epsilon}\left(\tilde{A}, \sigma_{1}\right) \stackrel{\rho_{1}}{\longrightarrow} W^{-\epsilon}(A, \sigma) \stackrel{\pi_{2}}{\longrightarrow} W^{\epsilon}\left(\tilde{A}, \sigma_{2}\right) \stackrel{\rho_{2}}{\longrightarrow} W^{-\epsilon}(A, \sigma) .
$$

Proof. See [4, Theorem 1.1].

Proposition 3.2. Let $(D, \sigma)$ be a central division algebra with involution over $k$. Then there exists a finite separable extension $M / k$ of odd degree such that the algebra with involution $\left(D_{M}, \sigma_{M}\right)$ over $M$ satisfies hypothesis $\left(^{*}\right)$.

Proof. See [3, Lemmas 3.3.1-3.3.3].

4. Witt groups in inseparable extensions. We keep the notation of the previous sections. In particular, $k$ is a field of characteristic $\neq 2$ and $(A, \sigma)$ is a finite dimensional central simple algebra with involution over $k$.

Theorem 4.1. Let $L$ be a purely inseparable extension of $k$. Then for $\epsilon= \pm 1$ the canonical homomorphism $r_{L / k}: W^{\epsilon}(A, \sigma) \rightarrow W^{\epsilon}\left(A_{L}, \sigma_{L}\right)$ is an isomorphism

We will prove Theorem 4.1 by induction on the degree of the algebra. We start by recalling two results which will be used in the first induction step. At least the first one is well-known, see for instance [8].

Proposition 4.2. Let $L$ be a finite purely inseparable extension of $k$. Then the canonical homomorphism $r_{L / k}: W(k) \rightarrow W(L)$ is an isomorphism.

Proof. Note that as $L / k$ is purely inseparable and $\operatorname{char}(k) \neq 2$, the degree of $L$ over $k$ is odd. This implies that $r_{L / k}$ is injective by Springer's theorem (or by Theorem 2.1). Let us prove that it is also surjective. As $L / k$ is purely inseparable, we have $L^{*} / L^{* 2} \simeq k^{*} / k^{* 2}$. On the other hand, every quadratic form over $L$ can be diagonalized. Hence every quadratic form over $L$ is extended from a quadratic form over $k$, and this concludes the proof of the proposition.

Let $K=k(\sqrt{d})$ for some $d \in k^{*}$, and let $\sigma: K \rightarrow K$ be the unique non-trivial $k$-linear involution of $K$. This is a unitary $K / k$-involution. Let $L$ be a purely inseparable extension of finite degree of $k$, and set $E=L(\sqrt{d})$. We then have:

Proposition 4.3. The canonical homomorphism $r_{L / k}: W(K, \sigma) \rightarrow W\left(E, \sigma_{L}\right)$ is an isomorphism.

Proof. It is well-known that there is an isomorphism of $W(L)$-modules

$$
W\left(E, \sigma_{L}\right) \simeq<1,-d>W(L)
$$

and an isomorphism of $W(k)$-modules $W(K, \sigma) \simeq<1,-d>W(k)$ which commute to base change (cf. for instance [9, Theorems 2.5.2 and 10.1.2]). As $r_{L / k}$ : $W(k) \rightarrow W(L)$ is an isomorphism by Proposition 4.2 , this implies that $r_{L / k}$ : $W(K, \sigma) \rightarrow W\left(E, \sigma_{L}\right)$ is an isomorphism too.

Using the following application of hermitian Morita theory, we will be able to suppose without loss of generality that $A$ is a division algebra. 
Theorem 4.4. Let $(A, \sigma)$ be a central simple algebra with involution over $k$ and let $\epsilon= \pm 1$. Then there exist a division algebra with involution $(D, \tau)$ over $k$ and $\epsilon^{\prime}= \pm 1$ such that the $W(k)$-modules $W^{\epsilon}(A, \sigma)$ and $W^{\epsilon^{\prime}}(D, \tau)$ are isomorphic. Moreover, for every field extension $L / k$, the $W(L)$-modules $W^{\epsilon}\left(A_{L}, \sigma_{L}\right)$ and $W^{\epsilon^{\prime}}\left(D_{L}, \tau_{L}\right)$ are isomorphic and the following diagram commutes:

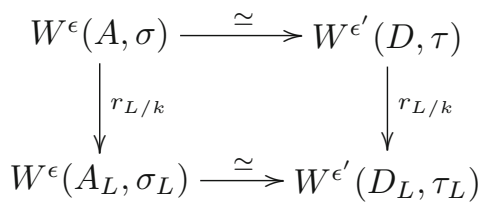

Proof. See [5, Chapter 1, Theorem 9.3.5.]

In the sequel for a field extension $M$ of $k$ we denote $R=M \otimes_{k} M$.

The following result will enable us to use separable odd degree base change.

Proposition 4.5. Let $f:(A, \sigma) \rightarrow(B, \tau)$ be a homomorphism of algebras with involution over $k$. Let $M$ be a separable extension of odd degree of $k$ such that the induced homomorphism $f_{M}^{*}: W^{\epsilon}\left(A_{M}, \sigma_{M}\right) \rightarrow W^{\epsilon}\left(B_{M}, \tau_{M}\right)$ is surjective and $f_{R}^{*}: W^{\epsilon}\left(A_{R}, \sigma_{R}\right) \rightarrow W^{\epsilon}\left(B_{R}, \tau_{R}\right)$ is a monomorphism. Then $f^{*}: W^{\epsilon}(A, \sigma) \rightarrow W^{\epsilon}(B, \tau)$ is an isomorphism.

Proof. By Theorem 2.2 we have the commutative diagram

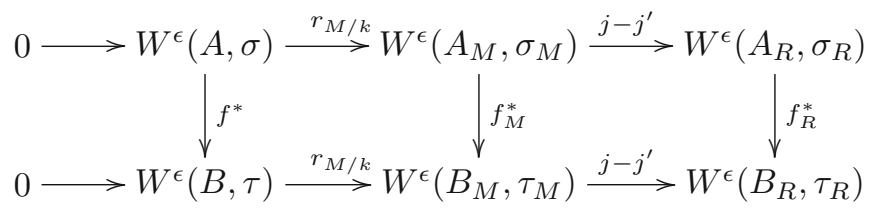

A diagram chase using the surjectivity of $f_{M}^{*}$, the injectivity of $f_{R}^{*}$ and the injectivity of $r_{M / k}$ shows that $f^{*}$ is surjective. The injectivity of $f^{*}$ follows from the injectivity of $f_{R}^{*}$.

Proof of Theorem 4.1. We prove the theorem by induction on the index of the algebra $A$. If this index is equal to 1 , then $A$ is a matrix algebra over $K$. By Morita theory (see Theorem 4.4) we can assume that $A=K$. Then either the involution is the non-trivial automorphism of $K / k$ or it is the identity and $K=k$. These cases are covered by Proposition 4.3, respectively Proposition 4.2, noting that $W^{-1}(K, \sigma) \simeq W(K, \sigma)$ (cf. [9, Remark 7.6.7]).

Let us assume that the index of $A$ is greater than 1. By 4.4, we can suppose that $A$ is a division algebra. Then there exists a finite, separable extension of odd degree $M / k$ such that $\left(A_{M}, \sigma_{M}\right)$ satisfies the hypothesis (*) of $\S 3$ (cf. 3.2). Using 4.5 , we may replace $A$ by $A_{M}$. Hence we have the exact sequence from Theorem 3.1. Let $L$ be a purely inseparable extension of $k$. It is easy to check 
that the following diagram is commutative:

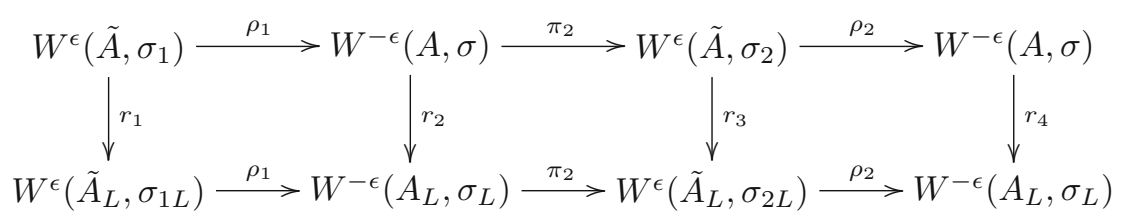

By the induction hypothesis, $r_{1}$ and $r_{3}$ are isomorphisms. In addition, $r_{4}$ is injective (cf. Theorem 2.1), so by the four-lemma we obtain that $r_{2}$ is surjective. Its injectivity clearly follows from Theorem 2.1. Replacing $\epsilon$ by $-\epsilon$, this completes the proof of the theorem.

Acknowledgements. D. A. Moldovan would like to thank Emmanuel Lequeu for many interesting and useful discussions.

\section{References}

[1] E. Bayer-Fluckiger, Unitary descent properties, J. Ramanujan Math. Soc. 24 (2009), 265-276.

[2] E. Bayer-Fluckiger and H. W. Lenstra, Jr., Forms in odd degree extensions and self-dual normal bases, Amer. J. Math. 112 (1990), 359-373.

[3] E. Bayer-Fluckiger, R. Parimala, Galois cohomology of linear algebraic groups over fields of cohomological dimension $\leq 2$, Appendix 2, by R. PARImala, R. Sridharan, and V. Suresh, Invent. Math. 122 (1995), 195-229.

[4] N. Grenier-Boley and M. Mahmoudi, Exact sequences of Witt groups, Comm. Math. 33 (2005), 965-986.

[5] M. Knus, Quadratic and hermitian forms over rings, Grundlehren der Math. Wiss. 294, Springer-Verlag, 1991.

[6] M. Knus et AL., The book of involutions, Coll. Pub. 44, Amer. Math. Soc., 1998.

[7] A. Rosenberg and R. Ware, The zero-dimensional Galois cohomology of Witt rings, Invent. Math. 11 (1970), 65-72.

[8] M. Rost, A descent property for Pfister forms, J. Ramanujan Math. Soc. 14 (1999), 55-63.

[9] W. Scharlau, Quadratic and Hermitian Forms, Grundlehren der Math. Wiss. 270, Springer-Verlag, 1985.

Eva BAyer-Fluckiger

École Polytechnique Fédérale de Lausanne, EPFL-FSB-MATHGEOM-CSAG,

Station 8, 1015 Lausanne,

Switzerland

e-mail: eva.bayer@epfl.ch 
Daniel Arnold Moldovan

École Polytechnique Fédérale de Lausanne, EPFL-FSB-MATHGEOM-CSAG,

Station 8, 1015 Lausanne,

Switzerland

e-mail: danielarnold.moldovan@epfl.ch

Received: 9 October 2010 